\title{
Zur Teratologie der Knochenfische, zugleich ein Beitrag zu deren Regeneration.
}

\author{
Von \\ Józef Nusbaum \\ in Lemberg. \\ (Zoologisches Institut der Universität.) \\ Mit Tafel $\mathrm{I}$.
}

Eingegangen am 21. März 1907.

Im vorigen Jahre erhielt ich aus einem Teiche in Opary bei Drobobycz (Ost-Galizien) ein junges Exemplar von Cyprinus carpio, welches $5 \mathrm{~cm}$ lang und $2 \mathrm{~cm}$ breit war, und einen teratologisch entwickelten Schwanzabschnitt des Körpers besaß.

Bald hinter dem After war der Körper verschmälert und bogenförmig am Hinterrande abgerundet, wobei die Rückenflosse und Afterflosse, welche eine ganz normale Zahl ron Flossenstrablen zeigten, in ihren hinteren Abschnitten gleicherweise bogenförmig gekrümmt waren, und den hinteren Rand des versehmälerten Körpers teilweise umgrenzten. . Aus der Mitte des hinteren Körperrandes trat ein weicher Anhang hervor, der sich nach hinten verschmälerte, seitwärts abgeplattet erschien, etwas mehr als $1 / 2 \mathrm{~cm}$ lang und an der Basis etwa $3 \mathrm{~mm}$ breit war; sein Hinterende war abgerundet. Der Anhang im ganzen war etwas nach links gerichtet. Das Fischchen war ganz gesund und munter, und lebte noch einige Wochen im Aquarium (Fig. 1).

Es unterliegt kaum einem Zweifel, daß diese teratologische Körperbildung durch eine unvollständige Regeneration eines zufällig verloren gegangenen, hinteren Körperabschnittes entstand, der sehr wahrscheinlich durch ein Raubtier abgebissen worden ist. Der Fall erscheint mir interessant in Anknüpfung an meine früheren Untersuchungen über die Regeneration bei den Teleostiern. 
Ich habe nämlich in einer $\left.{ }^{1}\right)$ meiner frihheren Arbeiten über die Regeneration der Knochenfische hervorgehoben, daß: 1) je älter das Tier ist, dem ein hinterer Körperabschnitt abgetrennt wurde, desto unvollständiger die Regeneration desselben vor sich geht und 2) je ein größerer hinterer Körperabschnitt abgetrennt wird, desto unvollständiger und mehr heteromorphisch der Regenerationsprozeß verläuft.

$\mathrm{Zu}$ solchen Resultaten gelang ich auf Grund. meiner Experimente an jugendlichen Exemplaren von Salmo irideus, die soeben aus den Eihüllen ausgeschlüpft sind oder 8-12 Tage alt waren.

Da die betreffenden Verhältnisse innig mit dem Falle, den ich zu beschreiben beabsichtige, zusammenhängen, und die diesbezügliche ausführliche Arbeit in der polnischen Sprache publiziert ist, erlaube ich mir zuerst einige Tatsachen aus dieser Arbeit mitzuteilen und einige Abbildungen aus derselben darzustellen, was desto mehr, meine icl, wünschenswert erscheint, da ich mich in der betreffenden vorläufigen Mitteilung, die den deutschen Lesern bekannt ist, nur mit einer einzigen halbschematischen Abbildung begnügte (Anatom. Anzeiger. 1903. Bd. XXII).

Die Fischchen, bei welchen die ganze Schwanzflosse bis zur Basis abgeschnitten wurde, ist die Regeneration total und geschieht auf einem dem embryonalen ganz ähnlichen Wege. Bei Exemplaren, deren Körper auf der Höhe der Analflosse durchgeschnitten wird, ist die Regeneration gleicherweise vollständig, aber der Weg derselben ist schon ein etwas differenter, da hier zuerst eine gemeinschaftliche anal-caudale Flosse (Fig. 2) erscheint, welche sich auf Kosten der noch übrig gebliebenen analen Flosse entwickelt und sich erst sekundär in eine anale und caudale differenziert. Und zwar in der zweiten, dritten Woche, oder etwas später beginnt der hintere und obere Teil dieser »anal-caudalen «Flosse sich zu differenzieren und bildet einen besonderen, dreieckigen Anhang, der dann weiter nach hinten wächst und die Anlage der Schwanzflosse bildet, in welcher keine selbständigen Anlagen der Flossenstrahlenträger hervortreten, sondern auf der Bauchseite des hintersten Teiles der Wirbelsäule als Differenzierungen der Parapophysen der betreffenden Wirbel hervortreten (Fig. 3).

Ganz andre Verhältnisse finden wir bei Individuen, welche auf der Höhe des Afters durchgeschnitten worden sind (es wurden Indi-

1) J. Nusbaum, Przyczynek do kwestyi regeneracyi ryb kostnoskieletowych (Beitrag zur Regenerationsfrage der Teleostier). Polnisch in der Zeitschrift "Kosmos». Lemberg 1903. Bd. 28. Mit 1 Tafel. 
viduen operiert gleich nach dem Verlassen der Eihüllen). Vier Wochen nach der Operation waren schon der neue After und die neue Urethralöffnung gebildet. Auch hier entstand zuerst eine gemeinschaftliche sanal-caudale * Flosse (Fig. 4, 5), die sich später in eine anale und caudale differenzierte. Als Flossenstrahlenträger der Analflosse bildeten sich hier im weiteren Verlaufe der Regeneration Knorpelblättchen nicht nur auf der unteren Seite der betreffenden Wirbel als Differenzierungen der Parapophysen, sondern auch auf der dorsalen Seite der zwei oder drei letzteren Wirbel als Differenzierungen der oberen Bogen. Hier regenerierte sich also die Schwanzflosse auf einem noch mehr heteromorphischen Wege als im vorigen Falle, was mit dem Abtragen einer größeren Körperpartie zusammenbängt. Die Abweichung von dem gewöhnlichen, normalen Typus besteht darin, daß 1) die anale und caudale Flosse anfangs miteinander zusammenhängen, und 2) daß die Flossenstrahlenträger sich nicht nur als Differenzierungen der Parapophysen der fünf letzten Wirbel, sondern auch als Differenzierungen der oberen Bogen der zwei oder drei letzten Wirbel entwickeln. Die Zahl der sich regenerierenden Metameren war hier vollständig.

Bei Exemplaren, bei denen ein noch größerer Körperabschnitt abgetragen wurde, und zwar welche quer zwischen dem After und der hinteren Grenze der Rückenflosse durehgeschnitten worden sind, war die Regeneration noch viel unvollständiger, da sich in keinem Falle die volle Zahl der abgetrennten Körpersegmente regenerierte, und in vielen Fällen nicht mehr als sechs oder sieben Körpermetameren sich gebildet haben, obwohl die Zahl der abgetrennten Körpersegmente 23 bis 25 betrug. Außerdem öffneten sich der neugebildete After und die definitive Urethralöffnung nicht, wie normal, auf der Bauchseite des Körpers, sondern direkt nach hinten auf dem Gipfel einer kleinen kegelförmigen Papille; manchmal öffnete sich nur der Darm auf dieser letzteren. Bei diesen Exemplaren bildete sich die anal-caudale Flosse am Hinterende des Körpers oberhalb der erwähnten Papille, bzw. oberhalb der beiden Öffnungen, also im Verhältnis zu diesen Öffnungen an einer ganz andern Stelle wie normal. Bei manchen Exemplaren entstand diese Flosse nur im unteren Teile des hinteren Körperrandes (Fig. 6) unterhalb der Muskelsegmente, oder sie reichte weiter nach oben bis zur Grenze zwischen den Rückenabschnitten und Bauchabschnitten der Muskelsegmente, oder endlich sie reichte noch weiter nach oben, so daß sie oberhalb der Analpapille längs des ganzen Hinterrandes des Körpers hervortritt. Im 
Mesenchymgewebe dieser Flosse erschienen knorplige Flossenstrahlenträger, und zwar ganz unabhängig von der Wirbelsäule; ich fand fünf oder sechs solche Flossenstrahlenträger, also eine viel geringere Zahl als in der normalen Analflosse, aber entsprechend eher der geringeren Zahl dieser Bildungen in der normalen Caudalflosse. AuBerdem wachsen in die Flosse Anlagen von Muskeln hinein, und zwar in der Zahl, die derjenigen der Knorpelstuicke entspricht, zwischen welche die ersteren eindringen. Die Muskeln sind Produkte von Myoblasten, welche sich vom Gewebe der sich regenerierenden Muskelsegmente lose abtrennen, aber keine Knospen der entsprechenden Myomere (Harrison) bilden, wie es im embryonalen Leben geschieht. Die sich auf diese Weise neubildende anal-caudale Flosse kann sich, wie erwähnt, eine größere oder kleinere Strecke längs des hinteren Körperrandes nach oben ziehen. Im ersteren Falle ahmt sie der Lage nach die typische Caudalflosse nach (Fig. 7). In dieser Pseudocaudalflosse entwickeln sich schon keine Parapophysen an der unteren Seite der letzten Wirbel der fast horizontal verlaufenden Wirbelsäule, was im Vergleich zum normalen Zustande, wo sich die Parapophysen zu starken Flossenstrahlenträgern der Schwanzflosse herausbilden, einen bedeutenden Unterschied darstellt. Hier besitzt diese Flosse ihre eignen Knorpelstiicke, die sich ganz unabhängig von der Wirbelsäule aus dem Mesenchymgewebe entwickeln. Die Pseudoschwanzflosse hat hier also den Charakter einer Analflosse und nur der Lage nach ahmt sie eine echte Caudalflosse nach, und spielt auch physiologisch die Rolle derselben. Es ist jedoch zu bemerken, daß öfters in den zwei oder drei letzten Wirbeln die unteren und oberen Bogen etwas größer sind, als in den mehr vorderen Wirbeln, was als eine gewisse physiologische Anpassung zur Verstärkung der Basis der Pseudocaudalflosse betrachtet werden muß.

Wenn ein jugendliches Exemplar von Salmo noch weiter nach vorn, und zwar auf der Höhe der dorsalen Flosse direkt hinter den paarigen Bauchflossen durchgeschnitten wird, dann spielt auch die Dorsalflosse eine bedentende Rolle in der Neubildung der Schwanzflosse. Die sich regenerierende Dorsalflosse bildet namentlich hinten einen Saum, der immer weiter das Hinterende des Körpers umgibt und als dorso-caudale Flosse funktioniert. Außerdem entsteht oberhalb der Analpapille die Anlage einer anal-caudalen Flosse, die ganz ähnlich derjenigen ist, welche wir im vorigen Falle beschrieben haben. Hier bleibt aber diese Anlage verhältnismäßig viel kleiner (Fig. 8). 
Querdurchschnitte von jugendlichen Forellen auf der Höhe der Dorsalflosse sind äußerst schwer zu bekommen, und zwar infolge der direkten Nachbarschaft der Dotterblase, welche dabei sehr leicht verwundet wird, weshalb ein großer Prozentsatz von operierten Exemplaren zugrunde geht. Ich konnte deshalb kein einziges Exemplar bekommen, bei welchem die dorsal-anale und anal-caudale Flosse vollständig miteinander zusammengewachsen wären. Das wäre viel leichter bei einigen andern Fischarten, z. B. beim jungen Hechte zu erzielen, wo die anale und dorsale Flosse in derselben Körpergegend eine gegentiber der andern liegt; bei der Forelle befindet sich aber bekanntlich die dorsale Flosse viel weiter vorwärts, als die anale. Beim jugëendlichen Hechte, der auf der Höhe der beiden Flossen quer durchgeschnitten würde, könnte man ohne Zweifel ein vollständiges Zusammenwachsen beider Flossen zu einer kontinuierlichen, gemeinschaftlichen pseudo-caudalen Flosse bekommen. Auf diese Weise entstanden sehr wahrscheinlich zwei von Prof. Bruvo HOFER beschriebene, teratologische Formen eines Hechtes, welchen wahrscheinlich ein Teil des Körpers hinten abgebissen worden ist (Allgemeine Fischerei-Zeitung. Nr. 1. 1901); die Abbildung eines dieser Exemplare (nach Hofer) gebe ich hier bei. Bei einem Exemplare befanden sich die dorsale und caudale Flosse im $\mathrm{Zu}-$ stande des Wachsens in der Richtung gegen das Hinterende des Körpers, aber es kam noch nicht zur Bildung einer einzigen Caudalflosse; man konnte noch dabei die nicht ganz geheilte Wunde am Hinterende konstatieren. Bei einem andern Exemplare (vgl. die beigegebene Abbildung. Fig. 9), welches HoFer zuerst beobachtete, war schon das Zusammenwachsen beider Flossen vollständig. In beiden Fällen waren die Flossenstrahlenträger ganz unabhängig von der Wirbelsäule; die Flosse am Hinterende des Körpers war also, wie Hofer ganz richtig annimmt, kein Produkt einer echten Candalflosse, sondern gehörte lediglich der dorsalen und analen Flosse an. Diese Beobachtung stimmt also vollkommen mit der meinigen an jugendlichen Forellen.

Was nun die teratologische Form des jungen Karpfen anbetrifft, welche ich hier beschreibe, so muß ich dieselbe in Anbetracht der oben angeführten Tatsachen aus einem Exemplare entstanden deuten, bei welchem ein Teil des Körpers direkt hinter der dorsalen und analen Flosse abgebissen wurde, das aber kein sehr jugendliches Individuum, sondern ein etwas älteres Exemplar war. Es scheinen mir folgende Gründe dafür zu sprechen: 1) eine außerordentlich 
große Differenz zwischen dem Durchmesser des Körpers und demjenigen des kleinen Anhanges (Regenerationskegels), 2) eine starke Entwicklung von Cutis und von Schuppen oder Schuppenpapillen in dem älteren Körperabschnitte und eine volle Abwesenheit von differenzierter Cutisschicht in dem Anhange, was dafür spricht, daß das Hinterende des Körpers in der Zeit abgetrennt wurde, wenn schon die Cutis samt den Schuppenpapillen ganz ausgebildet war, da in sehr jungen Stadien, was ich bei den sogleich aus dem Ei auisgeschlüpften Forellenindividuen beobachtete, gleichzeitig mit der Regeneration derEpidermis dieRegeneration der jungen, dünnen "Cutislamelle* samt der dieselbe bildenden Zellenschicht stattfindet, und den gleichen weiteren Veränderungen wie in alten Körperpartien unterliegt.

Mit einem Worte, alle diese Erwägungen führen uns zu einem sehr wahrscheinlichen Schlusse, daß dem Tier in der Gegend hinter der dorsalen und analen Flosse, und zwar in einem verhältnismäßig schon späteren postembryonalen Entwicklungsstadium, schon lange nach dem gänzlichen Verluste des Dottersackes durch irgendwelchen Zufall ein großer Körperabschnitt abgetrennt wurde. Die Regeneration war hier deshalb äußerst unvollkommen und verlief teilweise stark heteromorphisch, was zur Bildung einer teratologischen Form geführt hat.

Der erwähnte hintere Anhang unsres Fischchens hat eine etwas säbelförmige Gestalt; er ist, wie erwähnt, seitlich stark komprimiert, sein unterer Rand ist sehr schwach konvex, der obere dagegen am basalen Teile schwach konkav. Da er sich nach hinten hin verschmälert, zeigt er, von der Seite betrachtet, eine etwa kegelförmige Gestalt.

Die Querschnitte durch den Anhang zeigen uns, daß seine Epidermis mehrschichtig und mit einer basalen Schicht cylindrischer Zellen wie auch von vielen Schleimzellen, wie in der normalen Epidermis, versehen ist. Unter der Epidermis sieht man keine Cutisschicht, sondern das Innere des Anhanges ist von einem mesenchymatischen Gewebe ausgefüllt, welches ovale, spindelförmige und verästelte Zellen in einer homogenen Grundsubstanz eingebettet enthält und sehr stark vacuolisiert ist; die Epidermis liegt direkt diesem Gewebe an. In dem letzteren sieht man weder Muskelelemente, noch eine Verlängerang des Rückenmarkes; einzig und allein sind hier Skeletteile vorhanden, die schon gänzlich verknöchert sind und ziemlich stark veränderte Teile der Wirbelsäule darstellen. Von der Chorda dorsalis ist keine Spur zu sehen.

Das Knochengewebe ist nicht reich an Kalksalzen. In der Intercellularsubstanz des Gewebes, die eine feine Faserung zeigt, 
befinden sich in größerer Anzahl Knochenkörperchen, die in klęinen Lücken liegen; außerdem finden sich hier und da größere Höhlungen in der Mitte der einzelnen Knochenstiicke.

In dem basalen Teile des Anhanges fand ich vier Paare Knochenstücke, die ganz unabhängig voneinander in einem sehr lockeren, mesenchymatischen Gewebe eingebettet lagen, und an Querschnitten eine sichelförmige Gestalt zeigten, indem sie gegen die Hautdecke konvex, centripetalwärts dagegen konkar erschienen (Fig. 10).

Ich halte diese acht Knochenstiicke, welche mit dem letzten Wirbel des intakten Köperabschnittes innig zusammengewachsen sind, für Bildungen, die folgenden Wirbelteilen entsprechen, und zwar: die zwei unteren entsprechen den unteren Bogen, indem sie mit denselben des letzten Wirbels zusammengewachsen sind, die vier mittleren entsprechen dem Wirbelkörper, indem sie mit dem Körper des letzten Wirbels innig zusammenhängen, die zwei oberen endlich entsprechen den oberen Wirbelbogen.

Wenn, wie es normal in der ontogenetischen Entwicklung stattfindet, die Chorda samt Chordascheide entwickelt ist, dann treten die Knochenablagerungen kontinuierlich rings um die Chordascheide (ALBREcht, GoETTE) hervor; wobei diese Knochenablagerungen sich gewöhnlich ununterbrochen anf die Bogenbasen fortsetzen; in manchen Fällen (z. B. bei Coregonus nach ALвREch'r) stehen aber alle diese Knochenbeläge nicht in ununterbrochenem Zusammenhange, so daß die knöchernen Teile der distalen Abschnitte der Bogen durch Knorpelnähte von den basalen Teilen der Bogen und dem Knochenbelage des Wirbelkörpers getrennt bleiben.

Ich meine, daß in unserm Falle die Knochenstücke sehr wahrscheinlich ohne knorplige Vorbildung, sondern direkt aus dem umgebenden Bindegewebe entstanden zu deuten sind, da ich nirgends Reste vom Knorpelgewebe in dem Körperanhange gefunden habe. Hier entstanden obne Zweifel die Knochenteile der oberen und unteren Bogen ganz unabhängig von dem Knochenbelage der Wirbelkörper. Außerdem aber, im Mangel einer Chorda samt Chordascheide, bildete der demjenigen des Wirbelkörpers entsprechende Knochenbelag keinen kontinuierlichen Ring, sondern die vier erwähnten voneinander ganz unabhängigen, sichelförmigen Knochenstücke, die lose im mesenchymatischen Gewebe liegen.

Weiter nach hinten verändern sich die Verbältnisse der Knochenstücke, und zwar hinter dem ersten Drittel der Länge des Anhanges sind anstatt vier Paare ron Knochenstuicken nur zwei Paare derselben 
zu sehen. Die beiden unteren Paare wie auch die beiden oberen Paare der acht Knochenstiucke verbinden sich namentlich jederseits, so daß an jeder Seite zwei sichelförmige Knochenstücke, ein oberer und ein unterer, unter die Epidermis zu liegen kommen. Der Übergang ist ein allmählicher, und zwar findet man zuerst vier Knochenstiicke, deren jedes aus zwei Abschnitten, einem oberen und einem unteren, znsammengesetzt erscheint, wobei die Grenze zwischen diesen Abschnitten als longitudinal verlaufende Rinnen sowohl an der äußeren wie auch an der inneren Seite der Knochenstücke hervortreten, in der Richtung nach hinten allmählich seichter werden und endlich ganz versehwinden (Fig. 11).

Es ist interessant das Verhältnis des Epithels zu den erwähnten Knochenstücken; und zwar liegen diese letzteren mit ihren äußeren, konvexen Flächen dem Epithel so dicht an, daß dasselbe entsprechende Vertiefungen zeigt und zwischen den benachbarten Knochenstïcken jederseits Epithelleisten der basalen Zellenschichten bildet, die zwischen die Knochenstücke tief hineindringen. Mehr nach hinten, wo nur noch zwei Knochenstuicke jederseits vorhanden sind und jeder derselben die erwähnte seichte Rinne besitzt, ist die mittlere Epithelleiste ganz stark entwickelt und trennt die beiden Knochenstücke voneinander, die untere und obere Leiste ist dagegen schwächer und verschwindet endlich nach hinten.

In der hinteren Hälfte des Anhanges, wo nur vier Knochenstiicke ohne Spur von longitudinalen Rinnen und zwar ein Paar dorsaler und ventraler Elemente an Querschnitten zu sehen sind (Fig. 12), ist die erwähnte mittlere Epithelzellenleiste besonders stark entwickelt, sie ist hier breiter und tiefer, weshalb die beiden Knochenstïcke jederseits weit voneinander entfernt bleiben. Die beiderseitigen Epithelleisten dringen so tief in das unterliegende, mesenchymatische Gewebe hinein, $\mathrm{da} B$ sie sich fast berïhren und somit das ganze Innere des Anhanges in eine obere und untere Hälfte teilen. Die vier Knochenstücke, oder besser gesagt Knochenspangen, verlaufen longitudinal ganz unsegmentiert und kontinuierlich in der Richtung nach hinten, wobei sie immer dünner und niedriger werden, and bis zum Ende des Anhanges reichen, wo sie direkt dem Epithel des Hinterendes anliegen. Im hinteren Drittel des Anhanges ist das Epithel in der erwähnten Leiste wenigstens zweimal so dick, als an andern Stellen desselben.

Was das Mesenchymgewebe anbelangt, welches den Raum zwischen den Knochenspangen und dem Hautepithel erfüllt, so be- 
steht dasselbe, wie erwähnt, aus lose liegenden, spindelförmigen oder verästelten Zellen, welche hier und da in feine Fasern übergehen. Das Gewebe ist sehr stark vacuolisiert; die Gefäße verlaufen zum größten Teil in longitudinaler Richtung und bilden lange Schlingen, zwischen welchen durchwegs das Bindegewebe sich befindet.

Wie gesagt, dringen in den Anhang: weder Muskeln noch Rückenmark hinein. Das Rückenmark endet stumpf an dem Hinterende des intakt gebliebenen Körperabschnittes und ist hinten etwas kenlenförmig verdickt und abgerundet; es zeigt keine Spur von irgendwelehen regenerativen Prozessen. Mau sieht nur einzelne Äste der hintersten Rückenmarksnerven in den Anbang eindringen. Die Muskelsegmente sind in dem hintersten Teile des intakt gebliebenen Körperabschnittes, wo die Abrundung des Körpers stattgefunden hat, niedrig; etwas unregelmäßig untereinandergedrängt und vom Bindegewebe stark umhïllt; es ist daraus zu schließen, daß die verwundeten Muskelsegmente einer Heilung, aber keiner eigentlichen Regeneration unterlagen, was gleicherweise damit verknüpft ist, daß. die Verwundung in einem verhältnismäßig späten Entwicklungsstadium des Fischehens stattgefunden hat, da bei sehr jungen Fischexemplaren, die noch mit einer Dotterblase versehen sind, die Muskelsegmente sich wohl regenerieren, wie es meine diesbeziiglichen Untersuchungen an Forellen 1) nachgewiesen baben.

\section{Erklärung der Abbildungen.}

Tafel I.

Fig. 1. Photographische Aufnahme eines jungen Karpfens mit einem teratologischen Schwanzabschnitte des Kürpers. Links ein Hinterteil desselben Objektes mit kuinstlich abgehobenem Anhange, um zu zeigen, daß er etwas nach links gerichtet ist (fast natiirl. Größe).

Fig. 2. Ein Hinterteil des Körpers eines jugendlichen Salmo irideus, der den 9. Mai an der Höhe der Analfosse durchgeschnitten worden ist (fixiert den 10. Juni). p.o. Analflosse, a Anus. (Vergr.)

Fig. 3. Hinterteil eines Sagittalschnittes durch den jugendlichen Salmo ivideus, der auf der Höhe der Analflosse durchgeschnitten worden ist (operiert den 9. Mai, fixiert den 10. Juni). (Oc. 2 S. 3 ohne nntere Linse, Mikr. Merk. u. Ebex., mit Cam. gez.) og Anlage der definitiven Schwanzflosse, $k$ Flossenträger, $a$ Anus, $u$ Urethralöffnung, $n$ Riickenmark; ch Chorda dorsalis.

1) J. Nusbaum and s. Sidoriak, Beiträge zur Kenntnis der Regeneration nach küstlichen Verletzangen bei den äiteren Bachforellenembryonen. Archiv f. Entw.-Mech. Bd. X. 1900. 

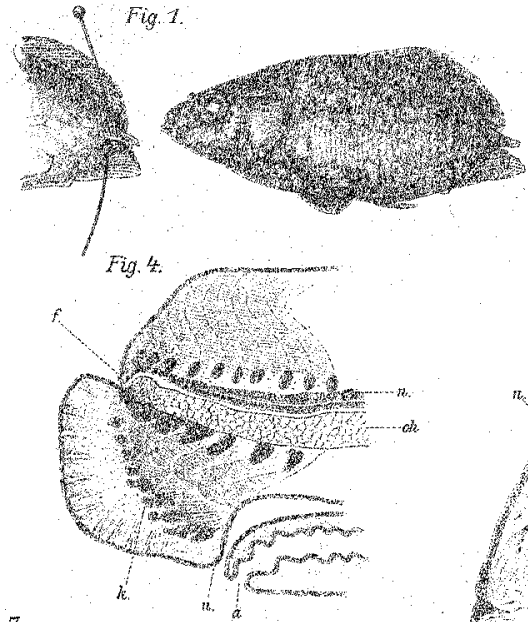

Fig. 7.
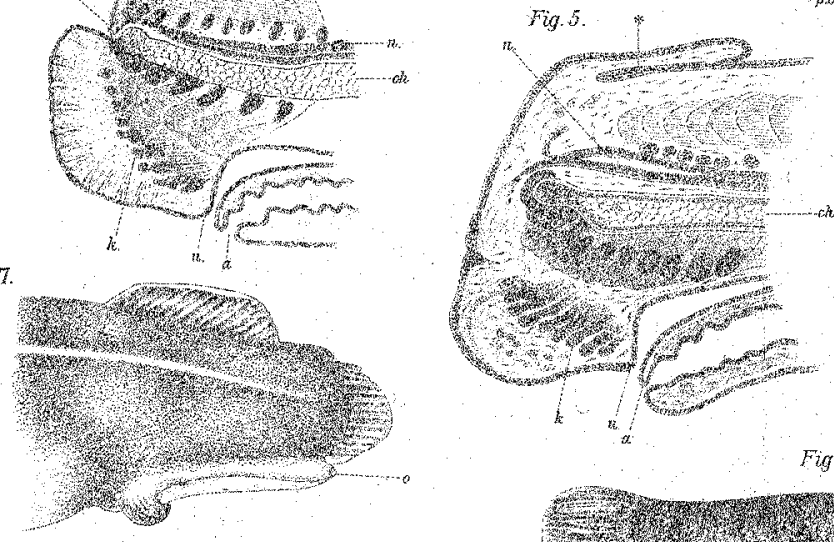

Fig. 9

Fig. 2.
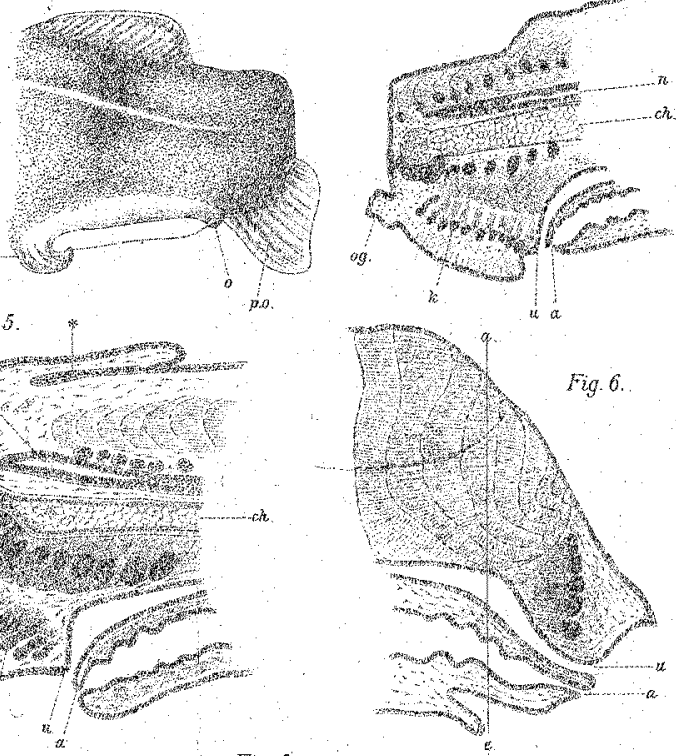

Fig

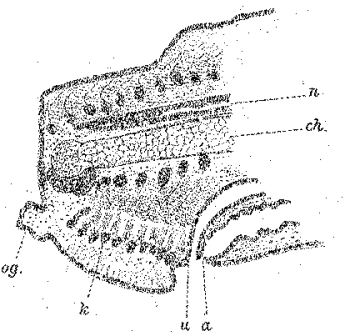

g. 6
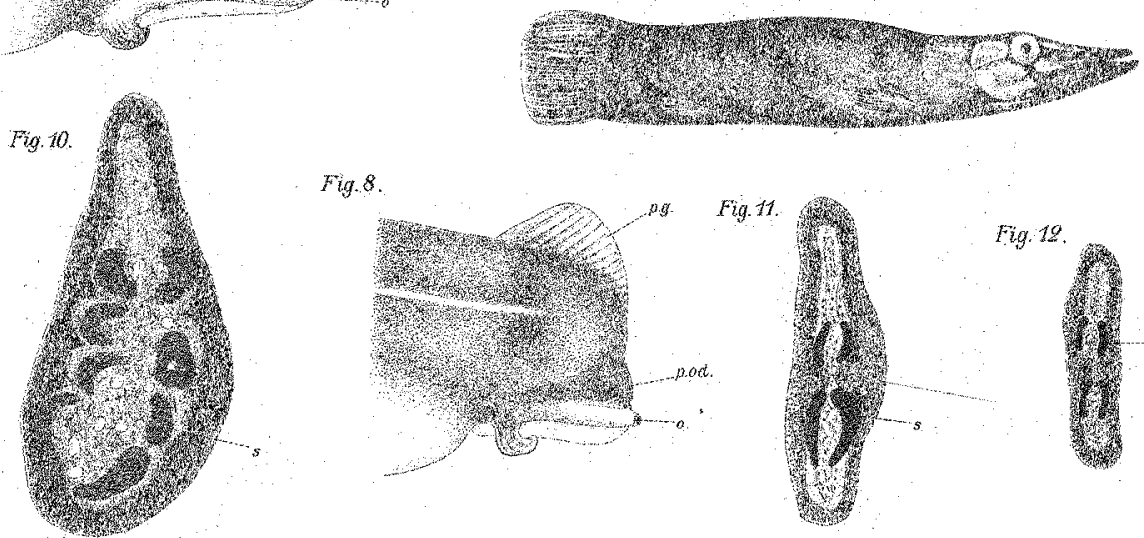

Fig. 8.

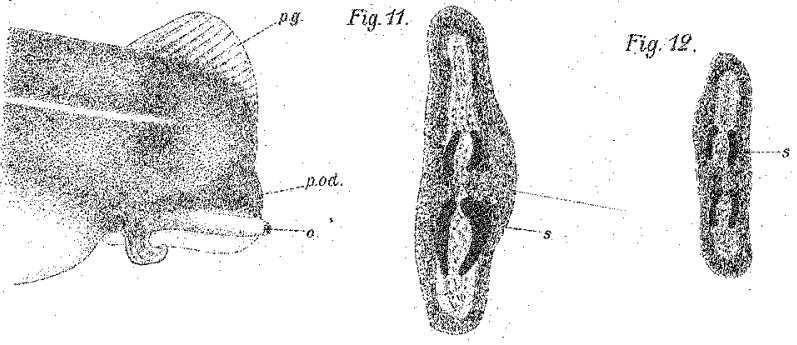


Fig. 4. Hinterteil eines Sagittalschnittes durch einen jugendlichen Salmo irideus, der anf der Höhe des Afters durchgeschnitten worden ist (operiert den 20. Mai, fixiert den 21. Juni). Vergrößerung und Buchstabenbezeichnung wie in Fig. 3.

Fig. 5. Teil eines Sagittalschnittes durch einen jugendlichen Salmo irideus, der auf der Höhe des Afters durchgeschnitten worden ist (operiert den 9. Mai, fixiert den 3. Juni). Vergrößerung und Buchstabenbezeichnnng wie in Fig. 3.

Fig. 6. Teil eines Sagittalschnittes durch einen jugendlichen Salmo irideus, der an der Höhe zwischen der Dorsalflosse und der Analflosse quer durehgeschnitten worden ist (operiert den 9. Mai, fixiert den 10. Juni). a Anus, $u$ Urethralöffnung. Vergrößerung wie in Fig. 3.

Fig. 7. Hinterteil eines jugendlichen Salmo irideres, der den 9. Mai operiert and den 10. Juni fixiert worden ist (quer durchgeschnitten zwischen der Dorsalund Analflosse). o Anus. (Vergr.)

Fig. 8. Hinterteil eines jugendlichen Salmo irideus, der den 20. Mai auf der Höhe der Dorsalflosse quer durchgeschnitten und den 21. Juni fixiert worden ist. p.g. Dorsalflosse, p.od. Anlage der Analflosse, o Anus. (Vergr.)

Fig. 9. Ein teratologiseh entwickelter Hecht mit einer Psendocaudalflosse, verkleinert (nach Brovo HoFER). Derselbe war wahrscheinlich anf der Höhe der Dorsal- und Anallosse verwundet.

Fig. 10. Querschnitt durch den basalen Teil des hinteren Anhanges des Karpfens (Fig. 1). $s$ Skeletteile. (Oc. 2 S. A. ohne untere Linse, ZeIss.)

Fig. 11. Querschnitt durch den mittleren Teil desselben Anhanges; dieselbe Vergrößerung und Bezeichnung.

Fig. 12. Querschnitt durch das hintere Drittel desselben Anhanges; dieselbe Vergrößerung und Bezeichnung. 